\title{
Structural organization of Trypanosoma cruzi
}

\author{
Wanderley de Souza \\ Laboratório de Ultraestrutura Celular Hertha Meyer, Instituto de Biofísica Carlos Chagas Filho, Universidade Federal do Rio de Janeiro, \\ Av. Carlos Chagas Filho 373 Bloco G subsolo, Cidade Universitária, Ilha do Fundão, Rio de Janeiro, RJ, Brasil, Diretoria de Programa, \\ Instituto Nacional de Metrologia, Normalização e Qualidade Industrial, Rio de Janeiro, RJ, Brasil
}

Since the initial description of Trypanosoma cruzi by Carlos Chagas in 1909, several research groups have used different microscopic techniques to obtain detailed information about the various developmental stages found in the life cycle of this intracellular parasite. This review describes the present knowledge on the organization of the most important structures and organelles found in the protozoan, such as the cell surface, flagellum, cytoskeleton, kinetoplast-mitochondrion complex, glycosome, acidocalcisome, contractile vacuole, lipid inclusions, the secretory pathway, endocytic pathway and the nucleus.

Key words: Trypanosoma cruzi - ultrastructure - electron microscopy

Since its initial description in 1909, the morphology of the various developmental stages of Trypanosoma cruzi has been the subject of intense investigation (Chagas 1909). A color drawing found in the original paper describing T. cruzi (Fig. 1) points to variations in the general shape of the protozoan and the presence of several structures that can be stained with Giemsa. It is important to point out from a historic point of view that $T$. cruzi was one of the first cells to be examined by Transmission Electron Microscopy (TEM) and has continued to be examined by almost all of the techniques developed over the last 50 years, as recently reviewed (De Souza 2008). In this review, I will briefly cover the main structures and organelles found in T. cruzi after 100 years of morphological descriptions. In view of space limitations, I will be necessarily telegraphic. However, I hope that the illustrations presented will speak for themselves.

Due to the ability to obtain in vitro axenic cultures, most of the studies on T. cruzi have been done with the epimastigote form. This is the proliferative developmental stage found in the intestine of the invertebrate host. For a better understanding of the description of the various structures and organelles found in $T$. cruzi, a schematic view based mainly on images obtained with the electron microscope was provided in De Souza (2008, Fig. 1). Below, I will describe the most relevant structural information of the following groups of structures/ organelles: the surface of the cell, flagellum, cytoskeleton and the metabolic organelles (that is, the kinetoplastmitochondrion complex, glycosomes, acidocalcisomes, lipid inclusions, components of the secretory pathway and components of the endocytic pathway).

Corresponding author:wsouza@biof.ufrj.br

Received 23 March 200

Accepted 29 May 2009

\section{The cell surface}

The surface of intracellular parasites such as T. cruzi initially interacts with cell surfaces in the vertebrate host and then with the membrane of the parasitophorous vacuole. It is also through the cell surface that the protozoan interacts with the epithelial intestinal cells of the invertebrate host. Therefore, it is important to understand the structural organization, the molecular array, the composition and the functional properties of all of the cell surface components of pathogenic protozoa. Here we will consider the cell surface to include the lipid bilayer and the associated components that face the extracellular medium and form the glycocalyx (also known as the cell coat or the surface coat). The molecules found on the cell surface of $T$. cruzi include the mucins, transialidase and the Tc85 family of glycoproteins, among others.

Scanning Electron Microscopy (SEM) is the most commonly used electron microscopy approach to analyze cell surfaces since it allows for the visualization of the entire surface of the cell. Recently, detailed information has been obtained with the use of a high resolution field emission scanning electron microscope (Sant'Anna et al. 2005). Using such an instrument, it is possible to visualize specialized areas of the cell surface such as the cytostome of epimastigotes, a structure involved in the uptake of macromolecules from the medium through a typical endocytic process. SEM has also been used to visualize the surface domains of the protozoan, especially by examining the localization of gold-labeled lectins using backscattered electrons. For instance, Fig. 2 shows that gold-labeled concanavalin A is preferentially localized to the cytostome region of the epimastigote forms of T. cruzi (Nakamura et al. 2005).

Conventional TEM of thin sections is the most common approach to analyzing the structural organization of cells. However, this technique only gives a general idea about the thickness and density of the glycocalyx. It has been shown that the trypomastigote form of $T$. cruzi presents a thinner surface coat when compared to the thick coat characteristic of the bloodstream form of Trypanosoma brucei (Vickerman 1969, De Souza \& Meyer 


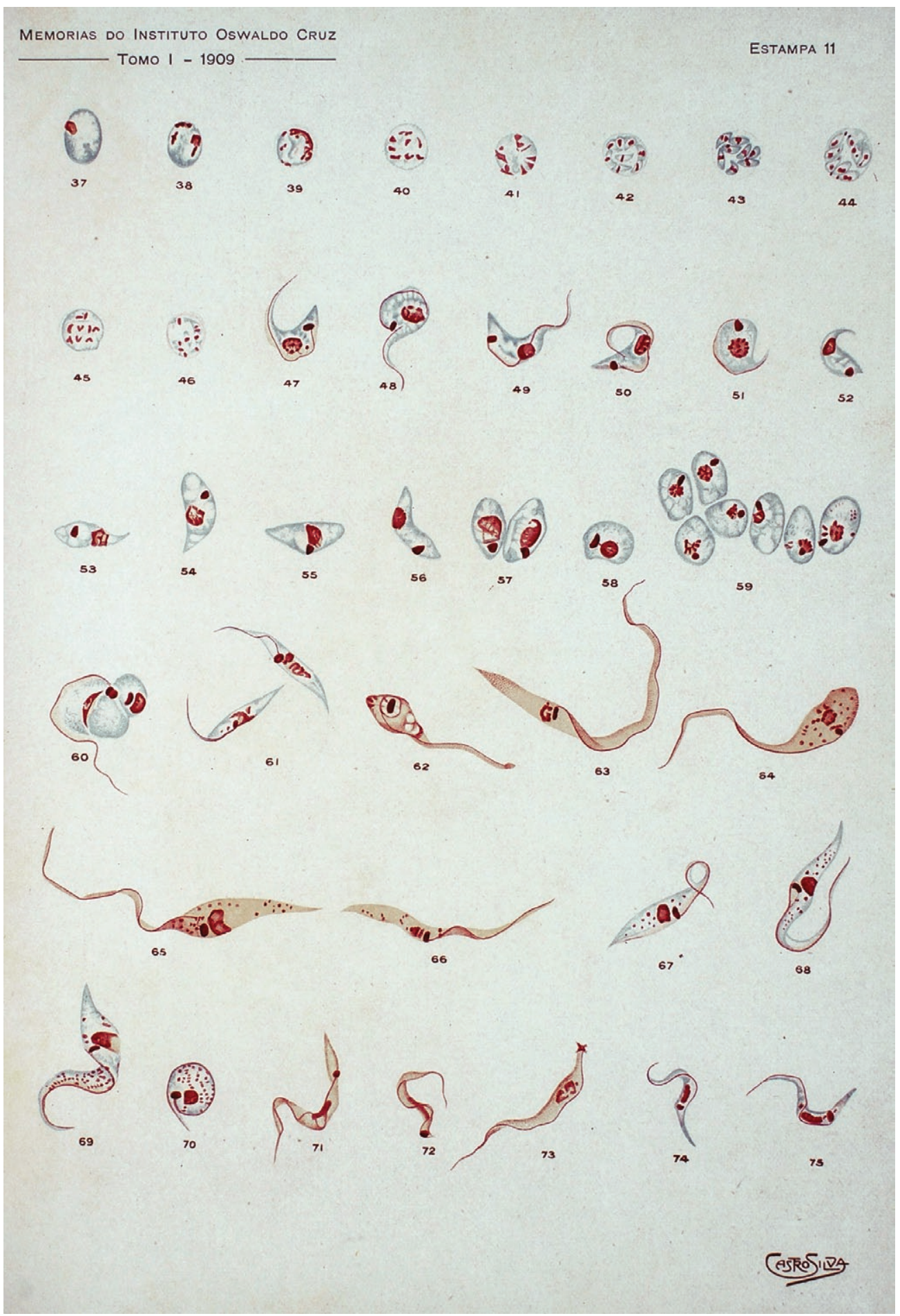

Fig. 1: reproduction of the original drawings of the first description of Trypanosoma cruzi by Carlos Chagas in 1909 where developmental stages found in both the invertebrate and invertebrate hosts were seen.

1975). The surface coat can be better visualized using cytochemical methods that detect carbohydrate-containing macromolecules (De Souza \& Meyer 1975) (Fig. 3) as well as with cationic dyes that bind to coat components and are intrinsically dense. Some examples of the latter are cationized ferritin particles and colloidal iron hydrox- ide particles; additionally, some dyes are osmiophilic, as in the case of ruthenium red (De Souza et al. 1977).

The quick-freezing, freeze-fracture, deep-etching technique allows for simultaneous observation of the protoplasmic face of the plasma membrane and a small area of the actual cell surface. Fig. 4 shows an extensive 


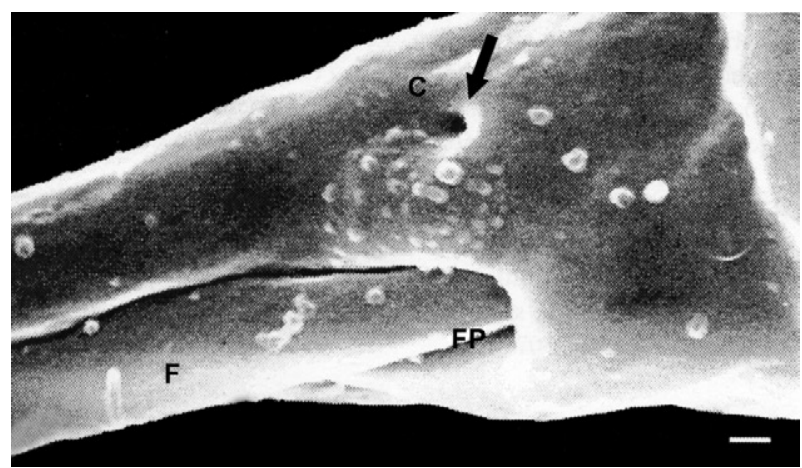

Fig. 2: high resolution scanning electron microscopy of an epimastigote form of Trypanosoma cruzi showing the cytostome (C), the flagellum (F) and the flagellar pocket (FP). The arrow points to the cytostome opening. Bar $=0.1 \mu \mathrm{m}$ (Nakamura et al. 2005).

area of the surface of $T$. cruzi that has been visualized using this approach. Another means for the visualization of large areas of the actual cell surface is the use of the fracture-flip technique. Fig. 5 shows an example obtained by this method from the surface of trypomastigote forms of T. cruzi (Pimenta et al. 1989). It is clear that the surface of trypomastigotes is very rugous. The rugosities probably correspond to proteins exposed on the protozoan surface. In the case of epimastigotes, the surface lining the cytostome region is more rugous than that covering the cell body and the flagellum.

The conventional freeze-fracture technique allows for the examination of the inner portion of the plasma membrane. Fig. 6 shows an example of an image obtained from an epimastigote form of T. cruzi (MartinezPalomo et al. 1976). The use of this technique revealed that the plasma membrane is not homogeneous in terms of the density and distribution of intramembranous particles (Fig. 6). Indeed, it is possible to identify at least three macrodomains of the membrane (Martinez-Palomo et al. 1976, De Souza et al. 1978, De Souza 2007): the cell body, the flagellum and the flagellar pocket. Each of these macrodomains possess specific microdomains such as (i) the flagellar necklace, localized at the basal portion of the flagellum; (ii) the zone of attachment of the flagellum to the cell body, where a linear array of intramembranous particles exist on both fracture faces of the flagellar membrane lining the adhesion region (Fig. 6) and (iii) the cytostome region, observed in epimastigote and amastigote forms of $T$. cruzi. The cytostome is an invagination of the plasma membrane followed by a few specialized microtubules that penetrate so deeply into the cell that they may reach the nuclear region. The opening of the cytostome can reach a diameter of up to $0.3 \mu \mathrm{m}$; however, it is significantly smaller in the deeper portion, the cytopharynx, which resembles a funnel. Freeze-fracture studies have shown that the membrane lining the cytostome is delimited by a palisade-like array of closely associated particles, corresponding to transmembrane proteins that remain unidentified thirty years after the initial description of this structure. The delim-

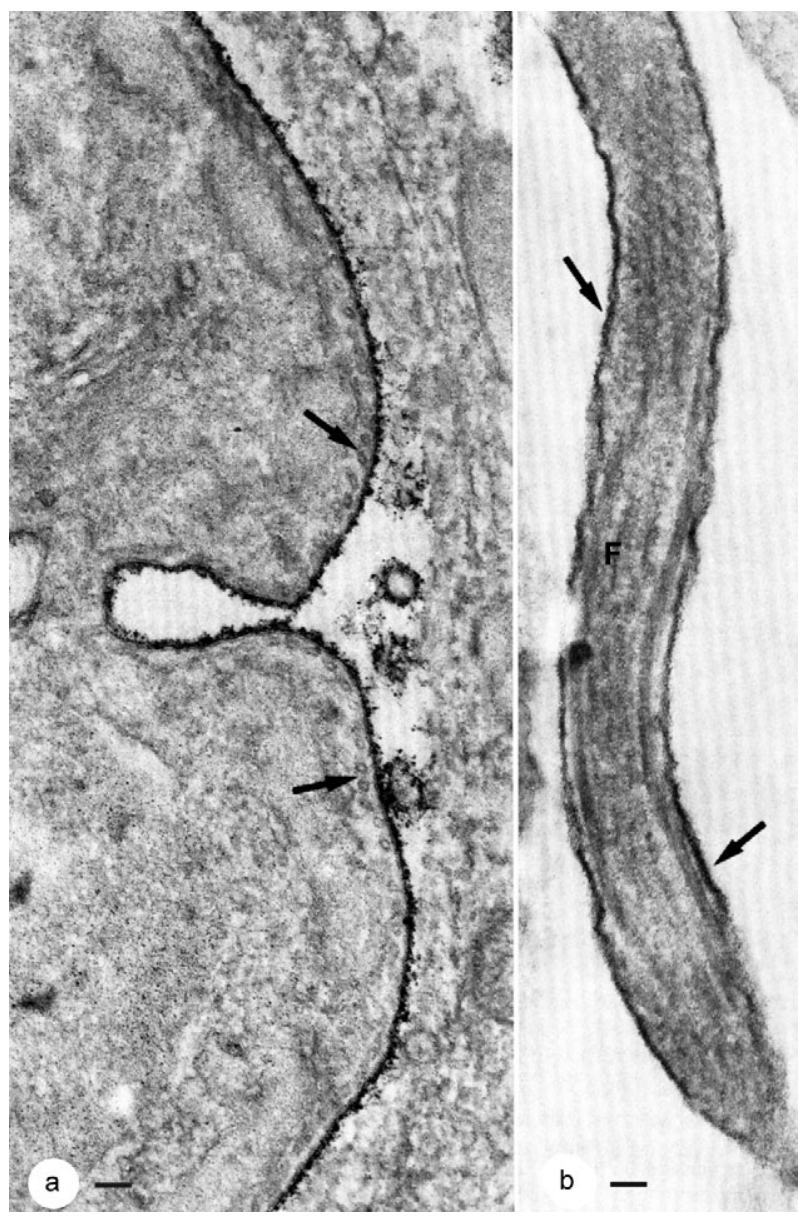

Fig. 3: localization of carbohydrate-containing molecules (arrows) on the surface of an intracellular amastigote form in division (a) and in the flagellum of a trypomastigote form (b). Reaction product, obtained using the periodic acid-thiosemicarbazide-silver proteinate technique, is seen in association with the thin surface coat. Bar $=100$ and $50 \mathrm{~nm}$, respectively (De Souza \& Meyer 1975).

ited area is nearly devoid of transmembrane proteins, appearing smooth in freeze fracture replicas. However, when the replicas are flipped, exposing the actual surface, the membrane lining the cytostome appears very rugous. As described above, this area contains a fibrillar material exposed on the surface. The combination of freeze fracture and cytochemical data indicates that the membrane lining the cytostome is rich in glycoconjugates that are not inserted in the membrane.

Freeze-fracture cytochemistry allows for the possibility of identifying components of the plasma membrane. The most successful example of this involves the use of digitonin and filipin to localize cholesterol and other $\beta$ hydroxy sterols. These compounds establish complexes with sterols and induce the appearance of either cylindrical or protruding structures, in the case of digitonin or filipin, respectively. These structures can be easily recognized in freeze-fracture replicas. Fig. 7 shows the distribution of filipin-sterol complexes in epimastigote forms of T. cruzi (Souto-Padron \& De Souza 1983). 


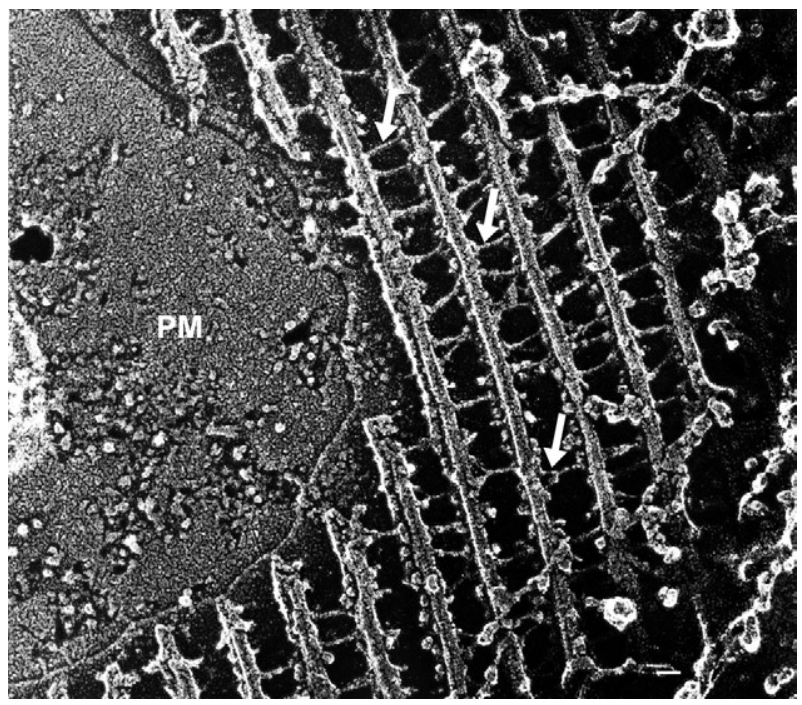

Fig. 4: deep-etching view revealing portions of the plasma membrane (PM) and the underlying layer of sub-pellicular microtubules showing connections between them (arrows). Bar $=100 \mathrm{~nm}$ (SoutoPadron et al. 1984).

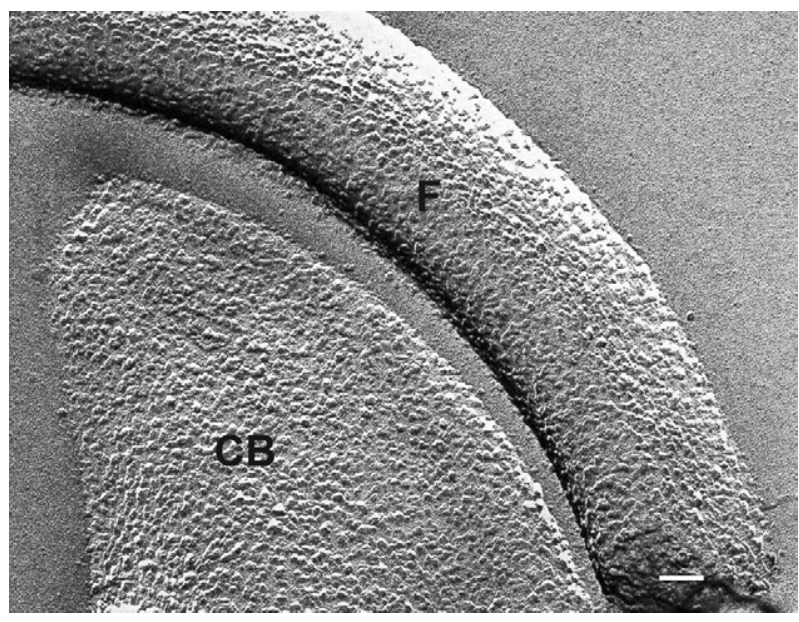

Fig. 5: fracture-flip of the surface of the cell body (CB) and flagellum (F) of the trypomastigote form of Trypanosoma cruzi. The suface appears with many rugosities. Bar = $100 \mathrm{~nm}$ (Pimenta et al. 1989).

\section{The flagellum}

A typical basal body located at the base of the unique flagellum was observed in the first thin sections examined of all T. cruzi developmental stages. Even in the socalled amastigote form, a short flagellum is observed. The flagellum shows the typical array of nine pairs of peripheral microtubule doublets and one central pair (Fig. 8). The first electron microscopy studies revealed the presence of an intriguing structure in the flagellum of trypanosomatids, which was designated as the paraxial or paraflagellar rod (PFR) due to its localization. The use of tannic acid in conjunction with glutaraldehyde

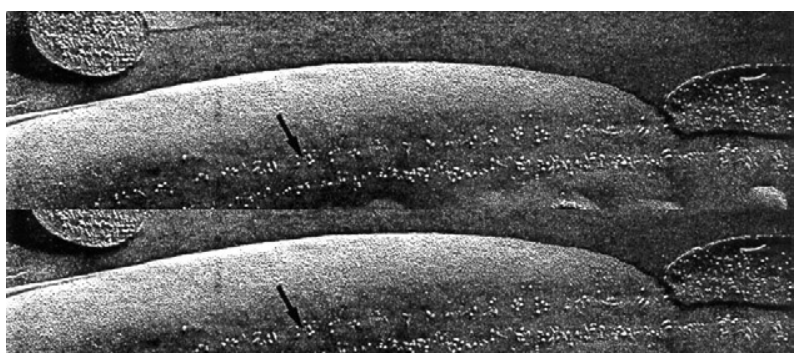

Fig. 6: freeze-fracture image revealing a special organization of intramembranous particles in the portion of the flagellar membrane involved in the adhesion of the flagelllum to the cell body. Bar $=100 \mathrm{~nm}$ (Martinez-Palomo et al. 1976).

significantly improved the preservation of this structure (Fig. 8). Subsequently, the use of quick-freezing, freezefracture, deep-etching and rotary replication (Farina et al. 1986, Souto-Padron et al. 1984) allowed the observation of several structures that were not visualized in thin sections (Fig. 8). It was seen that the PFR is made of a complex array of filaments linked to the axoneme. Two regions designated as proximal and distal were identified in the PFR. The proximal region is formed by two plates, while the distal region is formed by several plates. The plates are formed by an association of $25 \mathrm{~nm}$ and $7 \mathrm{~nm}$ thick filaments that are oriented at an angle of $50^{\circ}$ in relation to the major axis of the axoneme (Farina et al. 1986). The PFR is composed of a large number of proteins, most of which have not been yet characterized. However, two major proteins have been characterized in some detail. These proteins, known as PFR 1 and 2, have molecular weights of 73 and $79 \mathrm{kDa}$, respectively. They are highly antigenic and constitute targets for vaccine and diagnostic kit development. The available evidence indicates that the PFR is an essential structure for parasite survival (Bastin \& Gull 1999).

Atomic Force Microscopy (AFM) views of the flagellum revealed the presence of a furrow that separates the portion of the flagellum containing the axoneme from that containing the PFR (Rocha et al. 2006). Periodic structures were seen to be associated with the furrow (Fig. 9).

\section{The cytoskeleton}

Observation of thin sections of trypanosomatids by TEM revealed the presence of sub-pellicular microtubules distributed throughout the protozoan body, except in the flagellar pocket region. Using conventional fixation techniques, it is possible to see that the microtubules maintain a constant distance from each other (about 44 $\mathrm{nm}$ ) and from the plasma membrane (about $12 \mathrm{~nm}$ ). In favorable sections, it is possible to see some connections between these two structures. Profiles of the endoplasmatic reticulum (ER) can be seen in between and below the sub-pellicular microtubules (Pimenta \& De Souza 1985). When tannic acid is added to the glutaraldehyde solution, the protofilaments that make up the microtubules can be visualized (Baeta Soares \& De Souza 1979). 


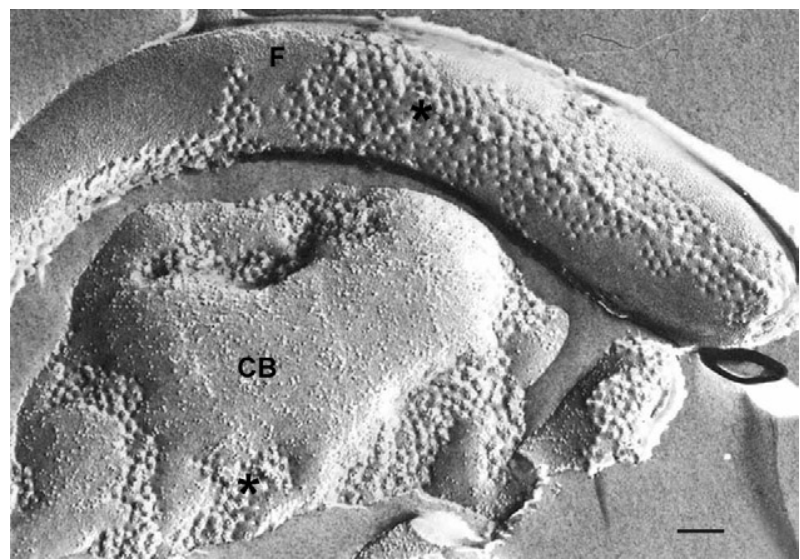

Fig. 7: freeze-fracture image of an epimastigote form of Trypanosoma cruzi incubated in the presence of filipin and then fixed. Filipin-sterol compexes (asterisks) can be seen both in the flagellar membrane (F) and in the membrane lining the cell body $(\mathrm{CB})$. Bar $=100 \mathrm{~nm}$ (SoutoPadron \& De Souza 1983).
One approach to observing the whole cytoskeleton of trypanosomatids is to lyse the protozoan on a water surface followed by critical point drying. Alternatively, cells can be lysed during drying, followed by adsorption to a formvar and carbon-coated grid and negative staining. With both techniques, it is possible to obtain images showing the helicoidal array of the microtubules. More recently, informative images of the organization of the sub-pellicular microtubules have been obtained using high resolution SEM. The use of stable cold field emission SEMs produces images with excellent resolution (Sant'Anna et al. 2005, De Souza et al. 2008).

Certainly the quick-freeze, freeze-fracture, deepetching and rotary replication technique is ideal for revealing details of cytoskeletal organization (SoutoPadron et al. 1984). This approach has yielded new information about the organization of sub-pellicular microtubules and the flagellum of trypanosomatids. In relation to the sub-pellicular microtubules, it is possible
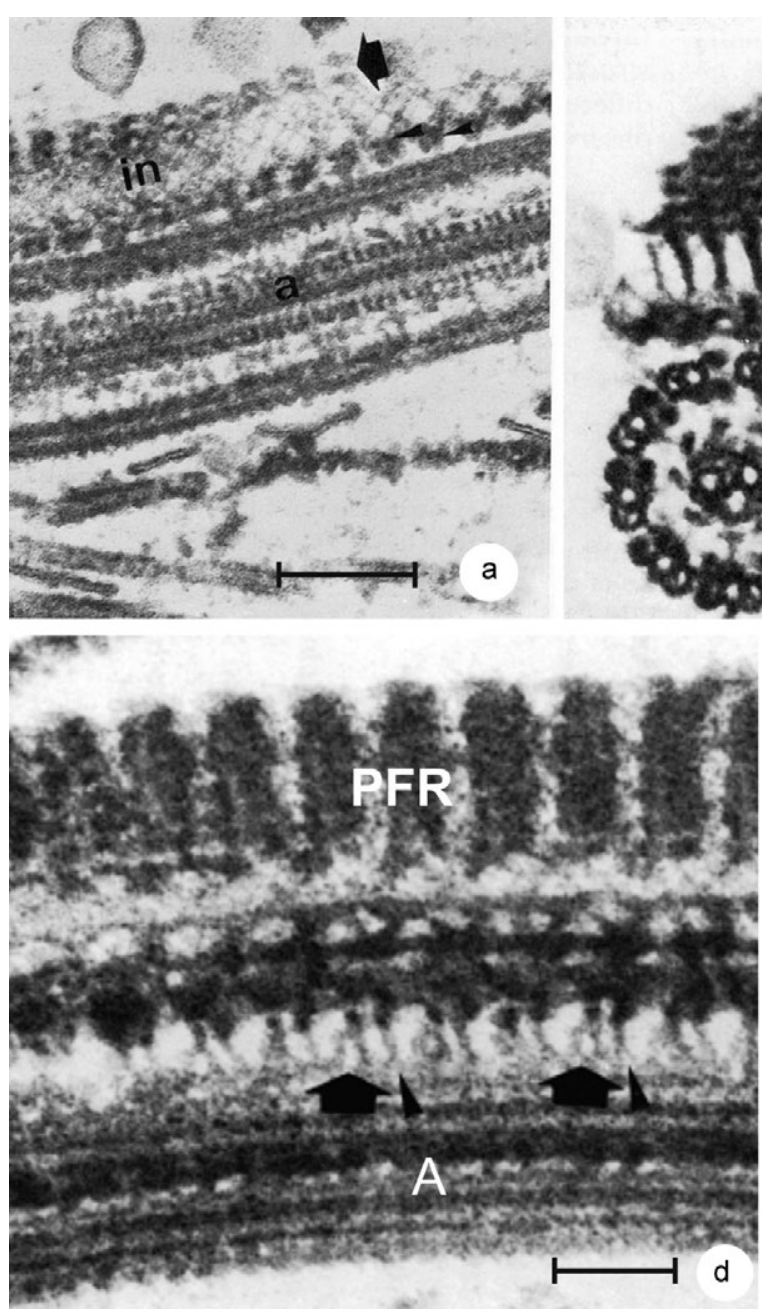
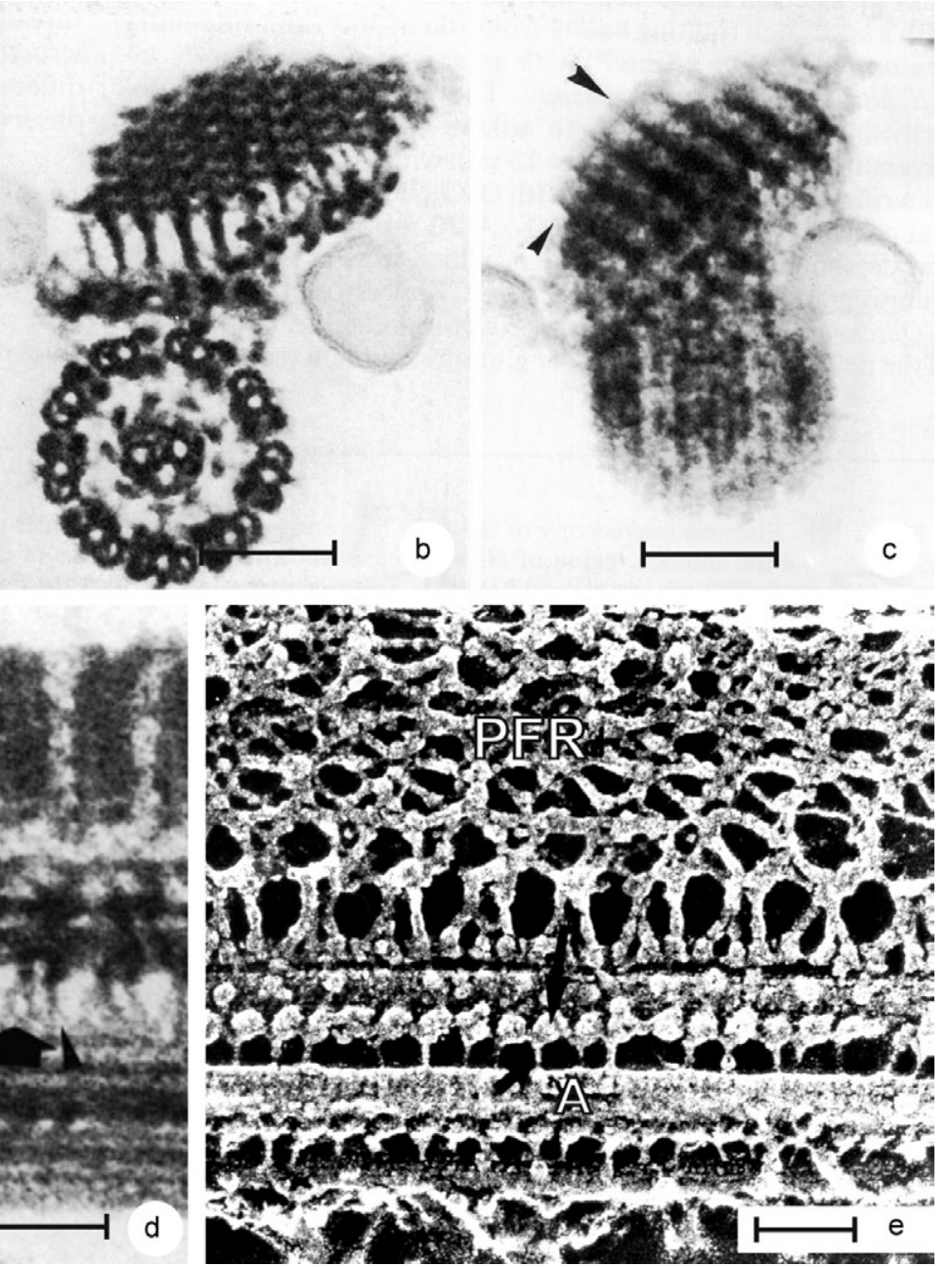

Fig. 8: different view of the flagellum of trypanosomatids as seen in longitudinal (a, d) and transversal (b, c) views of thin sections and replicas of quick-frozen, freeze-fractured, deep-etched and rotary repilicated samples (e). The axonemal (A) microtubules, as well as filaments which make the paraflagellar rod structure (PFR), can be seen. Bridges connecting the axoneme to the paraflagellar rod (arrows) and the plates which form the PFR are seen. Bar $=50(\mathrm{a}-\mathrm{c})$ and $100 \mathrm{~nm}(\mathrm{~d}, \mathrm{e})$ (Farina et al. 1986). 
to clearly see the filaments that connect microtubules to each other, to the inner portion of the plasma membrane and to profiles of the ER (Fig. 4).

Microfilaments have never been observed in the cytoplasm of $T$. cruzi. It was recently shown (Correa et al. 2007, 2008) that cytochalasin B treatment leads to morphological alterations in the cytoskeletal elements associated with the cytostome-cytopharynx complex, which is responsible for transferrin uptake. Comparative genomic analysis identified a potential role for an actinmyosin system in T. cruzi, as this protozoan presents an expanded myosin family and a CapZ F-actin capping complex in addition to an actin gene. It has been suggested that an actin-myosin system might function at the cytostome. Actin and actin-binding proteins have been characterized recently in T. cruzi (De Melo et al. 2008). TcActin was observed to exist in several patch-like cytoplasmic structures, present throughout the T. cruzi stages. Although the actin of T. cruzi has a similar structure to that found in higher eukaryotes, homology modeling has revealed fundamental differences, predominantly in the loops responsible for the oligomerization and interactions with actin-binding proteins. As a consequence, actin filaments have never been detected in T. cruzi.

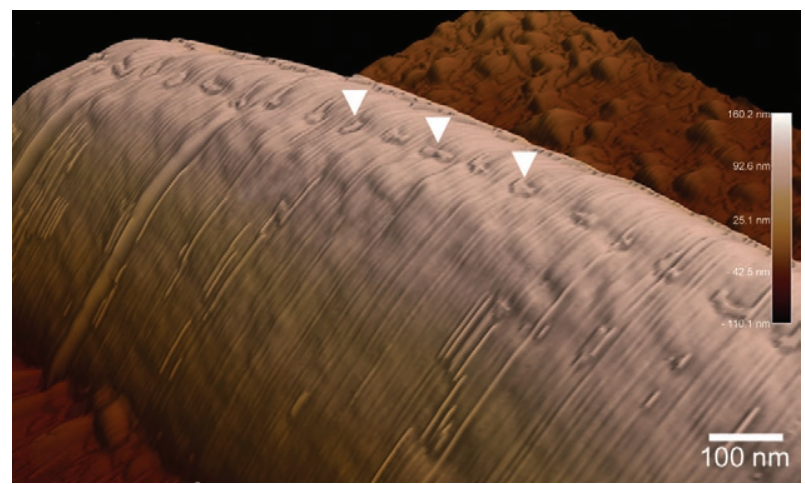

Fig. 9: AFM images of the flagellum region of slightly detergent-extracted cell. A furrow along the major axis of the flagellum is clearly seen. At high magnification of the furrow, periodically organized protrusions can be seen (arrowheads) (Rocha et al. 2006).

\section{The kinetoplast-mitochondrion complex}

Early studies using electron microscopy (Meyer et al. 1958) identified a dark, electron dense and slightly bent inclusion, corresponding to a structure known at that time as the kinetonucleus, which had been previously identified by light microscopy of stained cells. This structure is now known as the kinetoplast. It is situated close to the nucleus and its shape and structural organization vary according to the developmental stage of the protozoan. The remarkable molecular configuration of the DNA contained in kinetoplast was only revealed in the late 1960s and early 1970s (Shapiro \& Englund 1995, Lukes et al. 2002). It is now well established that trypanosomatids possess unique and highly ramified mitochondria. Subsequently, it was shown that the dense structure is made of a special type of DNA known as kinetoplast DNA (K-DNA). The first images obtained using thin sections clearly revealed the organization of the K-DNA and showed that it was localized to a specialized portion of the mitochondrion (Fig. 10). K-DNA is located within the mitochondrial matrix, perpendicular to the axis of the flagellum. The kinetoplast is always located close to the basal body. Filamentous structures connect the kinetoplast to the basal body (Fig. 11) (Souto-Padron et al. 1984, Ogbadoiry et al. 2003). Due to this connection, the position of the kinetoplast defines the cellular region in which the basal body is located and, consequently, the origin of the flagellum. Recently, a protein designated p166 was identified and shown to be located in between the K-DNA disk and the flagellar body (Zhao et al. 2008).

In addition to DNA electron microscopy, cytochemistry using the ethanolic phosphotungstic acid technique and ammoniacal silver (Souto-Padron \& De Souza 1978, 1979) demonstrated the presence of basic proteins in the kinetoplast, suggesting that these proteins may neutralize the negatively charged DNA molecules packed within this structure. More recently, evidence from biochemical and molecular experiments confirmed the presence of these basic proteins and indicated that histone-H1-like proteins participate in the condensation of K-DNA in Crithidia fasciculata and $T$. cruzi (De Souza \& Cavalcanti 2008).
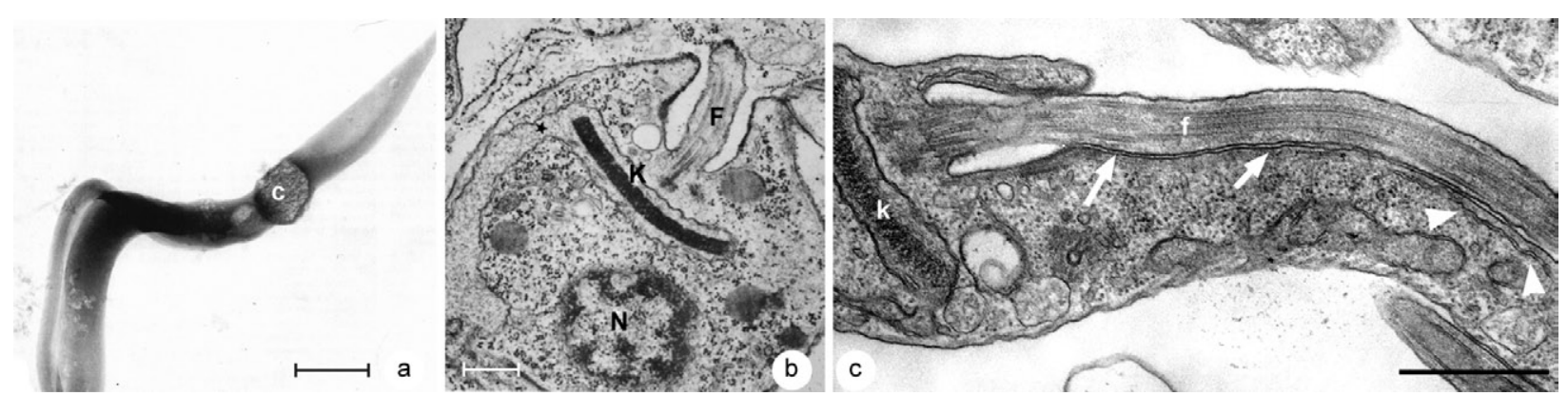

Fig. 10: different views of the kinetoplast and the general organization of the trypomastigote (a), amastigote (b) and epimastigote (c) forms of Trypanosoma cruzi. F: flagellum; K: kinetoplast; N: nucleus. The white arrows point to the area of adhesion of the flagellum to the cell body. White arrowheads point to profiles of the endoplasmic reticulum. Bars $=1 \mu \mathrm{m}$ (Rocha et al. 2006). 


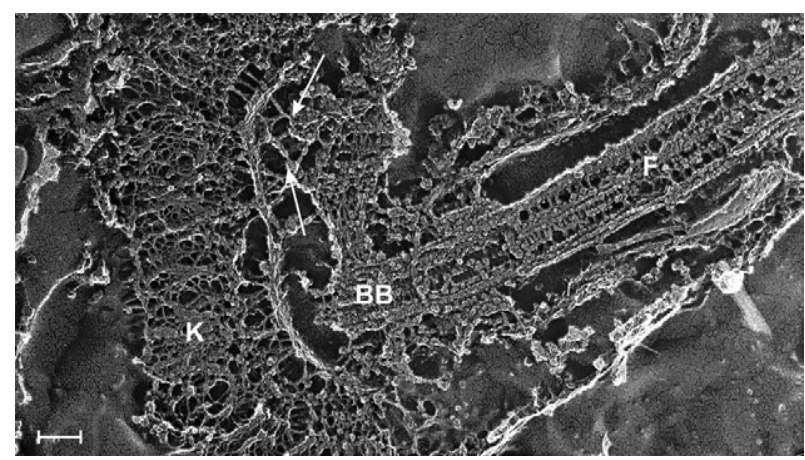

Fig. 11: anterior region of an epimastigote form of Trypanosoma cruzi as seen in a replica of quick-frozen, freeze-fractured, deep-etched and rotary replicated cells. The network of DNA molecules which make the kinetoplast $(\mathrm{K})$ is shown as well as filamentous structures (arrow) connecting the basal bodies (BB) to each other and to the mitochondrial membrane. The axonemal microtubules which make the flagellum (F) are also seen. Bar $=100 \mathrm{~nm}$ (Souto-Padron et al. 1984).

Two types of DNA rings are present in the kinetoplast, the minicircles and the maxicircles. There are several thousand minicircles, which range in size from about $0.5-2.5 \mathrm{~kb}$ (depending on the species) and a few dozen maxicircles, usually varying from 20-40 kb (Shapiro \& Englund 1995, De Souza \& Cavalcanti 2008). They encode guide RNAs that modify maxicircle transcripts by extensive uridylate insertion or deletion, a process known as RNA editing. Maxicircles are structurally and functionally analogous to the mitochondrial DNA in higher eukaryotes that encodes rRNAs and the subunits of respiratory complexes.

\section{The glycosome}

Examination of thin sections of several species of trypanosomatids revealed the presence of spherical structures with a homogeneous matrix, surrounded by a unit membrane. Initially, they were designated as microbodies, in analogy to similar structures that had been described in mammalian cells, particularly in hepatocytes. In the case of mammalian cells, it was shown that these structures contained catalase, an enzyme involved in the degradation of hydrogen peroxide formed in metabolic reactions. For this reason, they were termed peroxisomes. Using a cytochemical approach, catalase was also detected in the microbodies of $C$. fasciculata and in Leptomonas samueli (Opperdoes 1987). A major breakthrough in this area was the discovery that most of the glycolytic pathway takes place in this organelle (Opperdoes \& Borst 1977); thus it was eventually designated as the glycosome. In other cell types, glycolysis takes place in the cytosol. Since glycosomes found in the monogenetic trypanosomatids contain catalase, but those found in the digenetic ones do not, they are now considered to be a special type of peroxisome. Electron microscopy cytochemistry aimed at the detection of basic proteins, as previously described in the section regarding the kinetoplast, showed intense labeling of the glycosomes (Souto-Padron \& De Souza 1978). These observations prompted biochemical studies that demonstrated that the enzymes found in glycosomes have a higher isoelectric point than the cytosolic glycolytic enzymes found in other eukaryotic cells. In addition to catalase, the peroxisomes of mammalian cells contain more than 50 different enzymes involved in various metabolic pathways such as peroxide metabolism, $\beta$-oxidation of fatty acids, ether phospholipid synthesis and others. There is also evidence that additional metabolic pathways, such as carbon dioxide fixation (Opperdoes \& Cotton 1982), purine salvage and de novo pyrimidine biosynthesis, fatty acid elongation, isoprenoid biosynthesis and sterol biosynthesis (Opperdoes 1987), take place in the glycosomes of trypanosomatids, although they occur in the cytosol of other cells. The glycosome does not possess a genome. Therefore, all proteins found in it are encoded by nuclear genes, translated on free ribosomes and posttranslationally imported into the organelle.

\section{The acidocalcisome}

In the first observations of thin sections of T. cruzi and other trypanosomatids, vacuolar structures containing electron-dense deposits were found. These were designated as polyphosphate granules or volutin granules. Not until 1994 were these shown to be an organelle capable of transporting protons and calcium and it was henceforth named the acidocalcisome (Docampo et al. 2005). The appearance of this organelle depends on the methodology used to process the samples for electron microscopy. In conventional methods, most of the dense content may disappear, leaving only a small electrondense dot associated with the membrane lining the organelle (Fig. 12). However, when cryofixation is used, a much better preservation of acidocalcisome content can be achieved. Indeed, a homogenous electron-dense matrix was preserved when cells were quick frozen using a high-pressure freezing technique and submitted to freeze-substitution (Miranda et al. 2000). The best way to visualize the acidocalcisomes is to observe whole cells dried on a grid using an electron microscope with an energy filter, as shown in Fig. 12. Electron microscopy microanalysis has played a key role determining the ionic composition of the acidocalcisome. Using X-ray mapping, it has been shown that that acidocalcisomes contain calcium, phosphorous, sodium, potassium and zinc. In some trypanosomatids, iron has also been found (Miranda et al. 2004).

The acidocalcisomes are involved in functions such as (i) the storage of calcium, magnesium, sodium, potassium, zinc, iron and phosphorous compounds, especially inorganic pyrophosphate and polyphosphate, as determined by biochemical analysis and by X-ray microanalysis; (ii) $\mathrm{pH}$ homeostasis and (iii) osmoregulation, acting in close association with a contractile vacuole (Docampo et al. 2005).

\section{The contractile vacuole}

Although the presence of a contractile vacuole is very common in protozoa, there have been few reports on such structures in trypanosomes. Following initial light microscopy observations, a structure resembling 

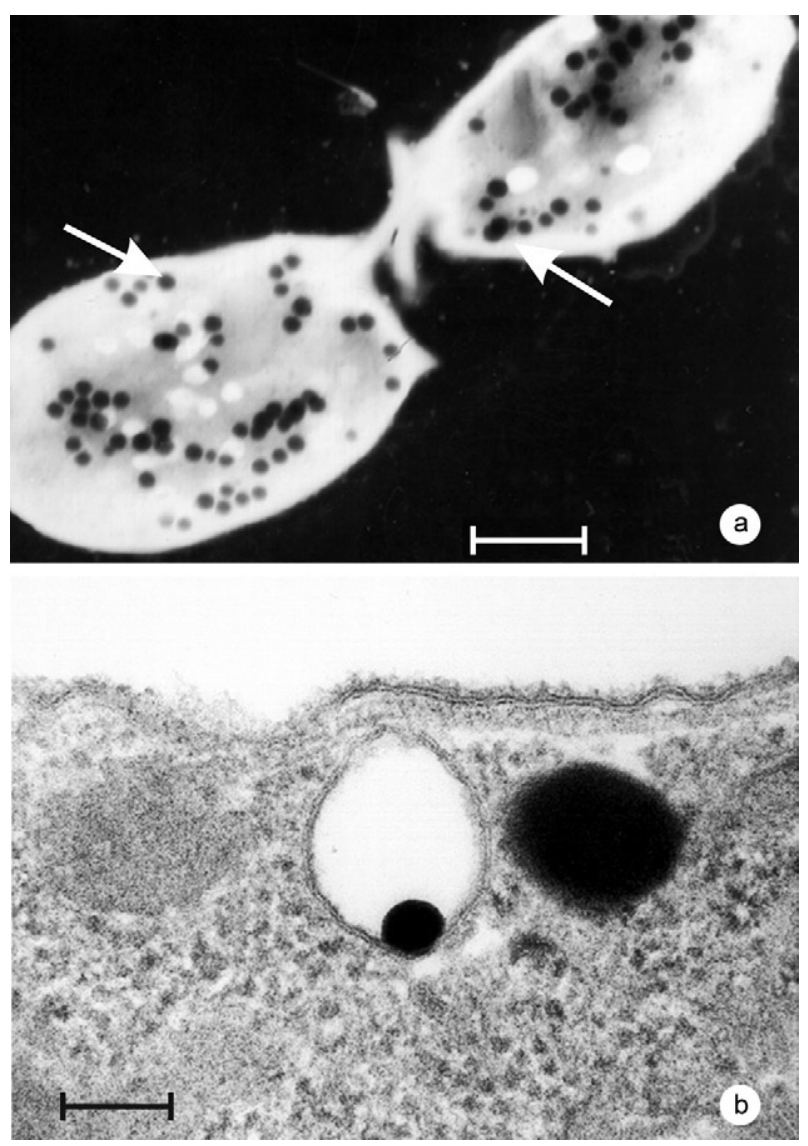

Fig. 12: acidocalcisomes of Trypanosoma cruzi as seen in whole cells (a) and in thin sections (b). When conventional methods of processing of samples for electron microscopy are used part of the dense material is released and the structure appears as partially empty. Bars $=1.0 \mu \mathrm{m}$ and $100 \mathrm{~nm}$, respectively (Miranda et al. 2004).

a contractile vacuole was characterized by electron microscopy. This structure is formed by several tubules connected to a central vacuole located close to the flagellar pocket (Linder \& Staehelin 1977). More recently, aquaporin, a protein involved in water transport, was identified in the epimastigotes of T. cruzi and shown to be localized to both acidocalcisomes and the contractile vacuole (Montalvetti et al. 2004). These structures seem to be involved in process of osmoregulation. It has been shown that fusion of acidocalcisomes to the contractile vacuole takes place via a process mediated by cyclic adenosine monophosphate (Rohloff \& Docampo 2008).

\section{Lipid inclusions}

Spherical structures with a variable diameter can be observed in the cytoplasm of T. cruzi. They are surrounded not by a typical unit membrane but by a phospholipid monolayer. Some are electron transparent, while others present a medium density. Most of them are highly contracted when the cells are fixed in an osmium tetroxide solution in immidazol buffer (Soares \& De Souza 1987). There is very little information about these structures.

\section{The secretory pathway}

The secretory pathway in $T$. cruzi is formed by the ER, Golgi complex and a system of vesicles that bud from the Golgi cisternae and migrate toward the flagellar pocket where they fuse and discharge their contents into the flagellar pocket. ER cisternae are seen around the nucleus and radiate towards all cell regions, especially the peripheral region where microtubules are located. Both rough and smooth ER cisternae can be seen. The Golgi complex is always located close to the flagellar pocket and is has morphology similar to that described in other cells. Labeling the Golgi complex with goldconjugated lectins revealed the presence of sugar-containing molecules, indicating its involvement in protein glycosylation, as has been reported for other eukaryotic cells. Rab7, a small GTPase involved in membrane trafficking, was also detected in the Golgi complex of trypanosomes (Araripe et al. 2004).

\section{The endocytic pathway}

For most eukaryotic cells, including pathogenic protozoa, endocytosis is the basic mechanism by which macromolecules are internalized. Endocytosed molecules are subsequently degraded in the endosomallysosomal system, providing important precursors for several key metabolic pathways. Trypanosomatids are highly polarized cells, and endocytic activity is restricted to the flagellar pocket and cytostome regions (De Souza et al. 2009). Studies of T. cruzi have shown that this protozoan exhibits certain peculiarities in its endocytic pathway that distinguish it from other cells. First, endocytosis only occurs when in the epimastigote form, which is found in the digestive tract of the invertebrate host and in axenic cultures maintained in vitro; endocytosis is low or absent in metacyclic or bloodstream localized trypomastigotes and in the intracellular amastigote forms. Second, epimastigotes have two sites of macromolecule ingestion: the flagellar pocket and a highly specialized structure known as the cytostome. Third, the cargo of the endocytic vesicles is delivered to unusual structures called reservosomes, which are localized at the posterior region of the protozoan. T. cruzi epimastigotes have a specialized plasma membrane region involved in endocytosis that starts at the opening of the cytostome and projects towards the flagellar pocket (Fig. 13). Quantitative analysis of the ingestion of gold-labeled macromolecules has shown that in epimastigote forms of T. cruzi, about $85 \%$ of gold particles associate with the cytostome (Porto-Carrero et al. 2000). Epimastigotes that are incubated with acridine orange, a weak base that concentrates in acidic compartments, accumulate dye in the cytopharynx, suggesting that this compartment has an acidic character. The presence of a P-type $\mathrm{H}^{+}$ATPase offers additional evidence of the acidic nature of the cytostome (Vieira et al. 2005). Following binding to the cytostome and flagellar pocket, macromolecules are rapidly internalized and appear in small endocytic vesicles that bud from the deeper regions of this structure (Soares \& De Souza 1991). Vesicles with a clathrin-like coat were first reported to bud from the Golgi complex. 

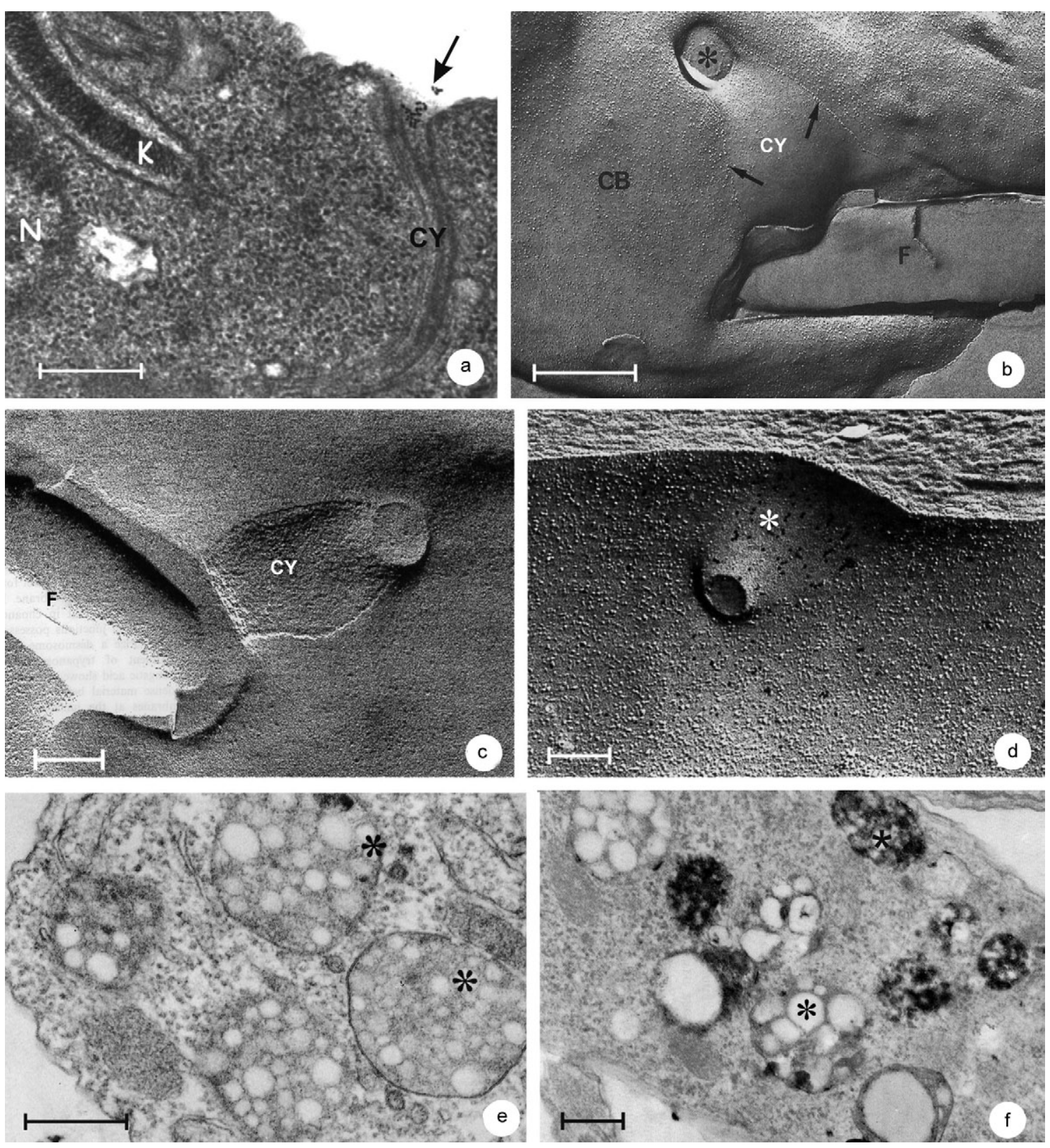

Fig. 13: a: thin section showing the cytostome (CY) of an epimastigote form of Trypanosoma cruzi in the initial step of ingestion of gold-labeled transferrin (arrow); b-d: freeze-fracture, fracture-flip and label-fracture views of the cytostome of epimastigotes of $T$. cruzi. The membrane lining the cytostome has few intramembranous particles, is intensely labeled with gold-concanavalin A and display surface projections; CB: cell body; e, f: reservosome of epimastigotes of $T$. cruzi appears as spherical structures with a matrix rich in some components which accumulate exogenous protein as horseradish peroxidase); F: flagellum; K: kinetoplast. Bars $=0.5 \mu \mathrm{m}$ (De Souza et al. 1978); Bars $=500,100 \mathrm{and} 100 \mathrm{~nm}$, respectively (Martinez-Palomo et al. 1976, Pimenta et al. 1989); Bar $=1.0 \mu \mathrm{m}$.

Corrêa et al. $(2007,2008)$ demonstrated that transferrin uptake is dependent on membrane cholesterol and on cytoskeletal elements that are associated with the cytostome. In silico analysis revealed the presence of clathrin, adaptin and clathrin self-assembly genes in its genome. Moreover, clathrin expression in T. cruzi was demonstrated by western blot using polyclonal antibodies raised against bovine clathrin heavy chain. TcClathrin has thus been localized to Golgi complex and flagellar pocket region. Curiously, agents that disturb receptormediated endocytosis do not impair transferrin uptake in epimastigotes (Correa et al. 2007, 2008). The fusion of endocytic vesicles with the tubule-vesicular network can be observed from the perinuclear region to the posterior tip of the protozoan. This tubular structure has been shown to be acidic by the use of acridine orange to probe the $\mathrm{pH}$ of intracellular compartments. The cargo pathway kinetics and $\mathrm{pH}$ suggest that this compartment may 
correspond to the epimastigote early endosome (EE). The spatial distribution and morphology of the EE have been detailed with a $3 \mathrm{D}$ reconstruction of a sequence of ultrathin sections.

A gene homologous to mammalian Rab5, an EE molecular marker, was cloned and sequenced and its expression has been measured in all T. cruzi developmental stages (Araripe et al. 2005). Its localization, however, has not been examined.

The major protease of $T$. cruzi, cruzipain, belongs to the cysteine protease family. It is very active in epimastigote forms and is concentrated in reservosomes. The enzyme is a glycoprotein synthesized in the ER-Golgi system in a proenzyme form and addressed to the endocytic pathway. The pro-peptide sequence is necessary and sufficient to drive cruzipain to reservosomes (Souto-Padron et al. 1990).

Macromolecules from the extracellular medium or from the ER-Golgi system are concentrated in structures known as reservosomes. These organelles are particularly interesting because they are found exclusively in the Schizotrypanum sub-genus, such as Trypanosoma vespertilionis, Trypanosoma dionisii and T. cruzi. Reservosomes are unique organelles that have a pivotal role in life cycle of $T$. cruzi. They were named for their unusual capacity to accumulate all of the macromolecules that are ingested by parasite through an endocytic process (Soares et al. 1992). In particular, T. cruzi epimastigotes specify a class of endocytic organelles, the reservosomes, whose main function is to store macromolecules, although they also concentrate lysosomal hydrolases. Thus, reservosomes are also considered the main site of protein degradation and regulation. Each epimastigote form has several reservosomes, mainly located in the posterior region of the cell (Fig. 13). They are surrounded by a unit membrane, with a mean diameter of $0.6 \mu \mathrm{m}$. They are usually globular but may appear asymmetrical. Ultrastructural cytochemical studies have shown that the electron-dense portion of the reservosome matrix is mainly composed of proteins while electron-lucent inclusions are likely to be lipids. Proteins, especially basic proteins, can be localized using the ethanolic phosphotungstic acid technique in cells that have been previously fixed in glutaraldehyde but not post-fixed in osmium tetroxide. Recently, the presence of internal vesicles both in isolated reservosomes and in situ was demonstrated using different TEM approaches (Sant'Anna et al. 2008). Long membrane profiles transversing the lumen of the reservosome were also observed. Another notable structure found was a rod-shaped electron-lucent structure bound by a membrane monolayer.

Using the DAMP technique to evaluate $\mathrm{pH}$ by electron microscopy, reservosomes were found to be comparable to mammalian late endosomes (pre-lysosomes), with a $\mathrm{pH}$ of 6.0, the presence of acid hydrolases and the lack of a lysosomal molecular marker (Soares et al. 1992).

Reservosomes have been described as an exclusive structure of epimastigote forms. While lipid and protein uptake has never been demonstrated in either trypomastigotes or amastigotes, intracellular organelles that share many reservosomal features were recently described in the mammalian stages of T. cruzi (Sant'Anna et al. 2008). Like reservosomes, these organelles are concentrated in the parasite's posterior region. They accumulate cruzipain, its natural inhibitor chagasin and serine carboxypetidase. They are acidic and have a P-type $\mathrm{H}^{+}$-ATPase. Interestingly, rod-shaped electron-lucent lipid bodies, similar to those that were recently characterized in the reservosome lumen, were also found in hydrolase-rich compartments in trypomastigotes and amastigotes. Collectively, these results indicate that these compartments are closely related. Nonetheless, they differ from reservosomes in the ability to store external macromolecules. Because of the low internal $\mathrm{pH}$ and the accumulation of lysosomal hydrolases, we have proposed that epimastigote reservosomes and the trypomastigote and amastigote organelles should be considered lysosomal-related organelles since they share fundamental properties with mammalian lysosomes.

Reservosomes have been considered the ultimate destination of macromolecules captured from the extracellular medium as well as the site where parasite major proteases accumulate. This organelle probably has lysosomal functions, as classical lysosomes have never been identified in $T$. cruzi. Nevertheless, aryl sulfatase activity, which is characteristic of lysosomes, has been detected inside small vesicles distributed throughout the cell bodies of epimastigotes and trypomastigotes (Adade et al. 2007). The digestive function of these compartments has not been addressed.

\section{The nucleus}

Initial observations revealed a nucleus enveloped by a typical porous membrane, condensed chromatin dispersed throughout the nucleoplasm and a nucleolus typical of the epimastigote, but not the amastigote or trypomastigote form (De Souza \& Meyer 1974, Solari 1995, Elias et al. 2001). It was also shown that the nuclear membrane remains intact during the entire division process, with the appearance of intranuclear microtubules, dispersion of the chromatin and the appearance of dense plates whose number varies according to the trypanosomatid species (Solari 1995). Still, there is no evidence that these plates correspond to chromosomes, which have thus far only been detected using a biochemical approach.

\section{Perspectives}

Electron microscopy will continue to provide important information on the structural organization of trypanosomes as new technological advances are made. I will comment here only on those approaches that are likely to be the most relevant over the next few years. First, there are very little data on the three-dimensional distribution of the various structures during the protozoan cell cycle. Certainly, important information can be obtained by three-dimensional reconstructions of the cell using both serial sections and electron microscopy tomography of epoxy-embedded sections. Second, more detailed information about some structures that can be isolated, such as the flagellum and kinetoplast, can be obtained using cryoelectron tomography. Third, electron microscopy analytical techniques using both X-ray 
mapping and electron energy loss spectroscopy and imaging will provide new information on the physiology of the organelles involved in ion transport. Fourth, immunocytochemistry of cryofixed cells will continue to add important information about the localization of new proteins discovered either by genomic or proteomic approaches. Fifth, AFM is a powerful technique for high resolution analysis of some structures, especially those that are part of the cytoskeleton, as was shown recently (Rocha et al. 2006) for structures within the flagellum. Sixth, high resolution SEM of the inner regions of the cell, exposed either by mechanical rupture or by freezefracture, has the potential to add a wealth of new information about the relationships between the various structures. Seventh, it will be necessary to use electron microscopy to improve the resolution of the information obtained using confocal light immunofluorescence microscopy of GFP-labeled proteins. A recent and important development in this area is the introduction of a new tag that can be detected using electron microscopy based on the use of the metal-binding protein metallothionein (Diestra et al. 2009). Finally, the various freeze-fracture techniques, especially in association with immunocytochemistry, will continue to be a powerful tool in the analysis of the structure of the cytoskeleton and various membranes.

\section{ACKNOWLEDGMENTS}

The author thanks several colleagues which have worked over the last 30 years in his laboratory and contributed with their work and enthusiasm to the progress of studies related with the cell biology of Trypanosoma cruzi as well as other members of the Trypanosmatidae family. Their contributions are indicated in the legend of figures used in this review as well as in the references.

\section{REFERENCES}

Adade CM, de Castro SL, Soares MJ 2007. Ultrastructural localization of Trypanosoma cruzi lysosomes by aryl sulphatase cytochemistry. Micron 38: 252-256.

Araripe JR, Cunha e Silva NL, Leal ST, De Souza W, Rondinelli E 2004. Trypanosoma cruzi: TcRAB 7 protein is localized at the Golgi apparatus in epimastigotes. Biochem Biophys Res Comm 321: 397-402.

Araripe JR, Ramos FP, Cunha e Silva NL, Urmenyi TP, Silva R, Leite Fontes CF, da Silveira JF, Rondinelli E 2005. Characterization of a RAB5 homologue in Trypanosoma cruzi. Biochem Biophys Res Commun 329: 638-645.

Baeta Soares T, De Souza W 1979. Fixation of trypanosomatids for electron microscopy with the glutaraldehyde-tannic acid method. Z Parasitenkd 53: 149-153.

Bastin P, Gull K 1999. Assembly and function of complex flagellar structures illustrated by the paraflagellar rod of trypanosomes. Protist 150: 113-123.

Chagas C 1909. Nova tripanozomiase humana. Estudos sobre a morfolojia e o ciclo evolutivo do Schizotrypanum cruzi n. gen. n. sp., ajente etiolojico de nova entidade mórbida do homem. Mem Inst Oswaldo Cruz 1: 159-218.

Corrêa JR, Atella GC, Batista MM, Soares M 2008. Transferrin uptake in Trypanosoma cruzi is impaired by interference on cytostome-associated cytoskeleton elements and stability of membrane cholesterol, but not by obstruction of clathrin-dependent endocytosis. Exp Parasitol 119: 58-66.
Corrêa JR, Atella GC, Menna-Barreto RS, Soares MJ 2007. Clathrin in Trypanosoma cruzi: in silico gene identification, isolation and localization of protein expression sites. J Euk Microbiol 54: 297-302.

De Melo LD, Sant'Anna C, Reis SA, Lourenço D, De Souza W, Lopes UG, Cunha e Silva NL 2008. Evolutionary conservation of actinbinding proteins in Trypanosoma cruzi and unusual subcellular localization of the actin homologue. Parasitology 135: 955-965.

De Souza W 2007. Macro, micro and nanodomains in the membrane of parasitic protozoa. Parasitol Int 56: 161-170.

De Souza W 2008. Electron microscopy of trypanosomes - A historical view. Mem Inst Oswaldo Cruz 103: 313-325.

De Souza W, Arguelo C, Martinez-Palomo A, Trissl D, GonzalesRobles A, Chiari E 1977. Surface charge of Trypanosoma cruzi. Binding of cationized ferritin and measurement of cellular electrophoretic mobility. J Protozool 24: 411-415.

De Souza W, Campanati L, Attias M 2008. Strategies and results of field emission scanning electron microscopy (FE-SEM) in the study of parasitic protozoa. Micron 39: 77-87.

De Souza W, Cavalcanti DP 2008. DNA-containing organelles in pathogenic protozoa: a review. Trends Cell Mol Biol 2: 89-104.

De Souza W, Martinez-Palomo A, Gonzáles-Robles A 1978. The cell surface of Trypanosoma cruzi: cytochemistry and freeze-fracture. J Cell Sci 33: 285-299.

De Souza W, Meyer H 1974. On the fine structure of the nucleus in Trypanosoma cruzi in tissue culture forms. Spindle fibers in the dividing nucleus. J Protozool 21: 48-52.

De Souza W, Meyer H 1975. Na electron microscopic and cytochemical study of the cell coat of Trypanosoma cruzi in tissue cultures. Z Parasitenkd 46: 170-187.

De Souza W, Sant'Anna C, Silva Filho NL 2009. Elecron microscopy and cytochemistry analysis of the endocytic pathway of pathogenic protozoa. Prog Histochem Cytochem, in press.

Diestra E, Fontana J, Guichad P, Marco S, Risco C 2009. Visualization of proteins in intact cells with a clonable tag for electron microscopy. J Struct Biol 165: 157-168.

Docampo R, De Souza W, Miranda K, Roheloff P, Moreno S 2005. Acidocalcisomes - conserved from bacteria to man. Nature Rev Microbiol 3: 251-261.

Elias MC, Marques-Porto R, Freymuller E, Schenckman S 2001. Transcription rate modulation through the Trypanosoma cruzi life cycle occurs in parallel with changes in nuclear organization. Mol Biochem Parasitol 112: 79-90.

Farina M, Attias M, Souto-Padron T, De Souza W 1986. Further studies on the organization of the paraxial rod of trypanosomatids. J Protozool 33: 552-557.

Linder JC, Staehelin LA 1977. Plasma membrane specialization in a trypanosomatid flagellate. J Ultrastruct Res 60: 246-262.

Lukes J, Guilbride DL, Votýpka J, Zíková A, Benne R, Englund PT 2002. Kinetoplast DNA network: evolution of an improbable structure. Euk Cell 1: 495-502.

Martinez-Palomo A, De Souza W, Gonzales-Robles A 1976. Topographical differences in the distribution of surface coat components and intramembranous particles. A cytochemical and freeze-fracture study in culture forms of Trypanosoma cruzi. J Cell Biol 69: 507-513.

Meyer H, Oliveira Musacchio M, Andrade Mendonça I 1958. Electron microscopy study of Trypanosoma cruzi in thin sections of infected tissue cultures and blood agar forms. Parasitology 48: 1-8. 
Miranda K, Benchimol M, Docampo R, De Souza W 2000. The fine structure of acidocalcisomes in Trypanosoma cruzi. Parasitol Res 86: 373-384.

Miranda K, Docampo R, Grillo O, De Souza W 2004. Acidocalcisomes of trypanosomatids have species-specific elemental composition. Protist 155: 395-405.

Montalvetti A, Rohloff P, Docampo R 2004. A functional aquaporin co-localizes with the vacuolar proton pyrophosphatase to acidocalcisomes and the contractile vacuole complex of Trypanosoma cruzi. J Biol Chem 279: 3867-3882.

Nakamura CV, Ueda-Nakamura T, De Souza W 2005. Visualization of the cytostome in Trypanosoma cruzi by high resolution field emission scanning electron microscopy using secondary and backscattered electron imaging. FEMS Microb Lett 242: 227-230.

Ogbadoiyi EO, Robinson DR, Gull K 2003. A high-order trans-membrane structural linkage is responsible for mitochondrial genome positioning and segregation by flagellar basal bodies in trypanosomes. Mol Biol Cell 14: 1769-1779.

Opperdoes FR 1987. Compartmentalization of carbohydrate metabolism in trypanosomes. Ann Rev Microbiol 41: 127-151.

Opperdoes FR, Borst P 1977. Localization of nine glycolytic enzymes in a microbody-like organelle in Trypanosoma brucei. FEBS Lett 80: $360-364$.

Opperdoes FR, Cotton D 1982. Involvement of the glycosome of Trypanosoma brucei in carbon dioxide fixation. FEBS Lett 143: 60-64.

Pimenta PF, De Souza W 1985. Fine structure and cytochemistry of the endoplasmic reticulum and its association with the plasma membrane of Leishmania mexicana amazonensis. J Submicroscop Cytol 17: 413-419.

Pimenta PF, De Souza W, Souto-Padron T, Pinto da Silva P 1989. The cell surface of Trypanosoma cruzi: a fracture-flip, replica staining label-fracture survey. Eur J Cell Biol 50: 263-271.

Porto-Carrero I, Attias M, Miranda K, De Souza W, Cunha e Silva NL 2000. Trypanosoma cruzi epimastigote endocytic pathway: cargo enters the cytostome and passes through an early endosomal network before storage in reservosomes. Eur J Cell Biol 79: 858-869.

Rocha GM, Brandao BA, Mortara RA, Attias M, De Souza W, Carvalho TM 2006. The flagellar attachment zone of Trypanosoma cruzi epimastigote forms. J Struct Biol 154: 89-99.

Rocha GM, Miranda K, Weissmuller G, Bisch PM, De Souza W 2007. Ultrastructure of Trypanosoma cruzi revisited by atomic force microscopy. Mic Res Tech 71: 133-139.

Rohloff P, Docampo R 2008. A contractile vacuole complex is involved in osmoregulation in Trypanosoma cruzi. Exp Parasitol 118: $17-24$

Sant'Anna C, Campanati L, Gadelha C, Lourenço D, Labati-Terra L, Bittencourt-Silvestre J, Benchimol M, Cunha e Silva NL, de
Souza W 2005. Improvement on the visualization of cytoskeletal structures of protozoan parasites using high-resolution field emission scanning electron microscopy (FESEM). Histochem Cell Biol 124: 87-95.

Sant'Anna C, Parussini F, Lourenço D, de Souza W, Cazzulo JJ, Cunha-E-Silva NL 2008. All Trypanosoma cruzi developmental forms present lysosome-related organelles. Histochem Cell Biol 130: $1187-1198$

Sant'Anna C, Pereira MG, Lemgruber L, de Souza W, Cunha e Silva NL 2008. New insights into the morphology of Trypanosoma cruzi reservosome. Microsc Res Tech 71: 599-605.

Shapiro TA, Englund PT 1995. The structure and replication of kinetoplast DNA. Ann Rev Microbiol 49: 117-143.

Soares MJ, de Souza W 1987. Ultrastructural visualization of lipids in trypanosomatids. J Protozool 34: 199-203.

Soares MJ, De Souza W 1991. Endocytosis of gold-labeled proteins and LDL by Trypanosoma cruzi. Parasitol Res 77: 461-468.

Soares MJ, Souto-Padron T, De Souza W 1992. Identification of a large pre-lysosomal compartment in the pathogenic protozoon Trypanosoma cruzi. J Cell Sci 102: 157-167.

Solari AJ 1995. Mitosis and genome partition in trypanosomes. Biocell 19: 65-84.

Souto-Padron T, Campetella OE, Cazzulo JJ, de Souza W 1990. Cysteine proteinase in Trypanosoma cruzi: immunocytochemical localization and involvement in parasite-host cell interaction. J Cell Sci 96: 485-490.

Souto-Padron T, De Souza W 1978. Ultrastructural localization of basic proteins in Trypanosoma cruzi. J Histochem Cytochem 26: 349-356.

Souto-Padron T, De Souza W 1979. Cytochemical analysis at the finestructural level of trypanosomatids stained with phosphotungstic acid. J Potozool 26: 551-557.

Souto-Padron T, De Souza W 1983. Freeze-fracture localization of filipin-cholesterolcomplexes in the plasma membrane of Trypanosoma cruzi. J Parasitol 69: 129-136.

Souto-Padron T, De Souza W, Heuser JE 1984. Quick-freeze, deepetch rotary replication of Trypanosoma cruzi and Herpetomonas megaseliae. J Cell Sci 69: 167-168.

Vickerman K 1969. On the surface coat and flagellar adhesion in trypanosomes. J Cell Sci 5: 163-193.

Vieira M, Rohloff P, Luo S, Cunha-e-Silva NL, de Souza W, Docampo $\mathrm{R} 2005$. Role for a P-type $\mathrm{H}^{+}$-ATPase in the acidification of the endocytic pathway of Trypanosoma cruzi. Biochem J 392: 467-474.

Zhao Z, Lindsay ME, Chowdhury AR, Robinson DR, Englund PT 2008. p166, a link between the trypanosome mitochondrial DNA and flagellum, mediates genome segregation. EMBO J 27: 143-154. 